Published in final edited form as:

Nat Biotechnol. 2008 March ; 26(3): 343-351. doi:10.1038/nbt1387.

\title{
Large-scale chemical dissection of mitochondrial function
}

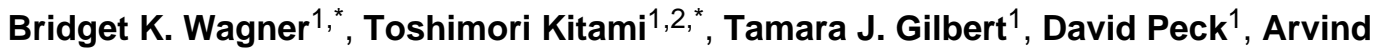 \\ Ramanathan ${ }^{1}$, Stuart L. Schreiber ${ }^{1}$, Todd R. Golub ${ }^{1,3}$, and Vamsi K. Mootha ${ }^{1,2,4}$ \\ ${ }^{1}$ Broad Institute of MIT and Harvard, Seven Cambridge Center, Cambridge, MA 02142 \\ ${ }^{2}$ Center for Human Genetic Research, Massachusetts General Hospital, Boston, MA 02114 \\ ${ }^{3}$ Department of Pediatric Oncology, Dana Farber Cancer Institute, Boston, MA 02115 \\ ${ }^{4}$ Department of Systems Biology, Harvard Medical School, Boston, MA 02446
}

\begin{abstract}
Mitochondrial oxidative phosphorylation (OXPHOS) is central to physiology and disease pathogenesis. To systematically investigate its activity and regulation, we performed a wide range of assays of OXPHOS physiology and nuclear and mitochondrial gene expression across 2490 chemical perturbations in muscle cells. Through mining of the resulting compendium, we discovered that: (1) protein synthesis inhibitors can de-couple coordination of nuclear and mitochondrial transcription; (2) a subset of HMG-CoA reductase inhibitors, in combination with nonselective beta-adrenergic receptor antagonists, can cause mitochondrial toxicity, providing clues into statin-associated myopathy; and (3) structurally diverse microtubule inhibitors stimulate OXPHOS transcription while suppressing reactive oxygen species, via a PGC-1a/ERRadependent mechanism, and thus may have utility in treating age-associated degenerative disorders. Our screening compendium is freely available and can be used as a discovery tool for understanding mitochondrial biology and toxicity, and identifying novel therapeutics.
\end{abstract}

\section{INTRODUCTION}

Oxidative phosphorylation (OXPHOS) is the core mitochondrial pathway responsible for ATP synthesis. The OXPHOS system consists of $\sim 90$ protein components, including all 13 of the proteins that are encoded by the mitochondrial genome (mtDNA)1. On time scales of seconds to minutes, mitochondrial ATP synthesis is regulated primarily by substrate availability and allosteric control2. During growth and development, however, transcription and translation of OXPHOS genes are carefully orchestrated between the nuclear and mitochondrial genomes to achieve sustained, metabolic adaptation. Over 50 mutations in the

\footnotetext{
Users may view, print, copy, and download text and data-mine the content in such documents, for the purposes of academic research, subject always to the full Conditions of use:http://www.nature.com/authors/editorial_policies/license.html\#terms

Correspondence: Vamsi K. Mootha, M.D., MGH Center for Human Genetic Research, 185 Cambridge Street CPZN 5-806, Boston, MA 02114, t: 617-643-3096, f: 617-643-2108, e: vamsi@ hms.harvard.edu.

These authors contributed equally to this work. Author contributions

V.K.M. conceived of and supervised the project. B.K.W., T.K., and V.K.M. designed the experiments and analyzed the data. T.A.G., A.R., and B.K.W. carried out phenotypic screening. D.P. and T.K. carried out GE-HTS experiments. S.L.S. and T.R.G. provided guidance on chemical screening and GE-HTS, respectively, and advised on analysis. B.K.W., T.K., and V.K.M. wrote the paper.
} 
mtDNA and nuclear genome have been linked to rare but devastating inborn errors of OXPHOS metabolism3. Moreover, quantitative declines in OXPHOS activity and efficiency have been linked to nearly all age-associated degenerative diseases, including type 2 diabetes mellitus4,5,6. Hence, understanding OXPHOS function and regulation, particularly within the context of the entire cell, will have important implications for human disease.

Traditional approaches to studying energy metabolism in the mitochondrion have focused either on the kinetics of ATP synthesis in isolated mitochondria, or on transcriptional control of mitochondrial components. For instance, classic bioenergetic studies using isolated mitochondria in combination with chemical inhibitors 2 focused on the acute regulation of mitochondrial activity, ignoring the cell's ability to respond and adapt over longer time scales. Many of the chemical reagents used in these studies were incompatible with cellular or animal studies, making it difficult to extend their relevance in vivo. More recently, molecular studies have shed light on the transcriptional control of OXPHOS7, though relatively little is known about how these regulatory programs are coupled to other cellular processes.

Our goal was to combine physiologic and genomic profiling of intact cells, to probe OXPHOS function and regulation in response to thousands of small-molecule perturbations. A systematic mapping between physiology and gene expression may shed light on how the nuclear genome and mtDNA are coordinated under different cellular conditions to maintain energy homeostasis. Large-scale perturbation-based approaches have been used successfully to study cell growth8, gene expression9,10, and tumorigenesis 11 , but to our knowledge, no previous studies have combined cell-based, physiological readouts with genomic profiling. The advantage of our integrated approach is that it provides a richer description of cellular mitochondrial state reporting on more stable changes in the organelle, which can be useful to study its longer-term adaptations.

Here, we report the construction of a mitochondrial screening compendium and its application to problems in mitochondrial biology, drug toxicity, and therapeutics. Our compendium is freely available and should provide a framework for understanding how the activity and regulation of mitochondrial OXPHOS are integrated within the context of the entire cell.

\section{RESULTS}

\section{Complementary profiles of OXPHOS physiology and gene expression across 2490 chemical perturbations}

We developed high-throughput assays in 384-well format for cell viability, mitochondrial physiology, and gene expression (Fig. 1, Methods). We used differentiated C2C12 murine myotubes, a cell line that has been used extensively to study mitochondrial biogenesis and bioenergetics 12. Cell viability was measured using a calcein dye13. We monitored mitochondrial physiology by probing four parameters related to OXPHOS. These included the JC1 assay, which measures mitochondrial membrane potential $\left(\Delta \Psi_{\mathrm{m}}\right) 14$; the MTT assay, which measures mitochondrial dehydrogenase activity 15; a luciferase-based assay for cellular ATP levels16; and a fluorescent probe $\left(\mathrm{CM}-\mathrm{H}_{2} \mathrm{DCFDA}\right)$, which measures reactive 
oxygen species (ROS), a by-product of OXPHOS17 (see Methods). We also developed an immunofluorescence-based assay for cytochrome $c$ protein content (see Methods).

To complement these physiological assays, we also performed gene expression-based highthroughput screening (GE-HTS)18,19 to profile the nuclear and mtDNA OXPHOS transcripts (see Methods). GE-HTS is a facile, high-throughput method by which dozens of transcripts can be simultaneously quantitated. It is a multiplexed, PCR strategy that combines ligation-mediated amplification with multi-colored bead detection to identify and quantitate transcripts of interest (see Supplementary Fig. 1 online). We adapted GE-HTS to profile simultaneously all $13 \mathrm{mtDNA}$-encoded OXPHOS (mtOXPHOS) transcripts as well as 12 nuclear-encoded OXPHOS (nuOXPHOS) transcripts (Supplementary Fig. 1 online). These 12 nuOXPHOS transcripts include representatives from all five OXPHOS protein complexes and were selected because they capture virtually all of the variation in gene expression exhibited by the entire OXPHOS repertoire, as assessed by analysis of over 5000 genome-wide microarrays (data not shown). Of note, our GE-HTS assay also monitored transcripts that tend to be anticorrelated to OXPHOS or are invariant across many conditions as assessed by microarrays, and thereby assist in data analysis (see Methods). Together, our GE-HTS assay faithfully "tags" the expression of the entire OXPHOS system. Moreover, because the expression of OXPHOS genes is so correlated, measuring multiple transcripts increases the signal-to-noise ratio with which we can detect subtle effects of compounds4.

We performed the viability, physiology, and gene expression assays in duplicate in differentiated $\mathrm{C} 2 \mathrm{C} 12$ myotubes following 48-hour treatment with each of 2,490 compounds. Our chemical library consists of known bioactives and is enriched in FDA-approved drugs. Using a scoring algorithm dependent upon the distribution of mock-treated (DMSO) wells20,21, we arrived at a normalized score for each assay in each well (Supplementary Table 1 online). In our compendium, we have included data from our screen for cytochrome $c$ protein expression, though we excluded it from subsequent analyses since the coefficient of variation was high. Correlation analysis indicated that our remaining readouts (one for viability, four for OXPHOS physiology, and one for OXPHOS gene expression) provide complementary information (see Supplementary Fig. 2 online).

The resulting compendium is freely available to the public and is useful for dissecting mitochondrial function (Supplementary Table 1 online). Unlike traditional methods, it enables us systematically to track how changes in nuclear and mitochondrial OXPHOS gene expression are coupled to mitochondrial physiology over thousands of perturbations. We used this approach to explore three problems focused on mitochondrial biology, drug toxicity, and novel therapeutics.

\section{Cross-talk between nuclear and mitochondrial OXPHOS gene expression}

First, we used the compendium to identify the cellular signals involved in coordinating nuclear OXPHOS (nuOXPHOS) and mtDNA OXPHOS (mtOXPHOS) transcription. OXPHOS gene expression from the two genomes must be tightly coupled to maintain energy homeostasis in the mitochondrion22,23. Moreover, OXPHOS expression can change in human diseases, though often, it is unclear whether the changes are primary or reactive, and how these changes map to cellular physiology4,24-26. We therefore focused on the 
relationship between nuOXPHOS and mtOXPHOS transcripts across the chemical perturbations. As expected, the majority of compounds influence the two sets of genes in a coordinated manner (Fig. 2a). However, we identified some compounds that decouple the coordination between these two genomes (Fig. $2 \mathrm{~b}$ and Supplementary Table 2 online), a subset of which we confirmed with follow-up dose response curves and RT-PCR analysis (Fig. 2c). Specifically, we discovered that the eukaryotic protein synthesis inhibitors emetine, anisomycin, and cycloheximide preferentially increase nuOXPHOS expression, implying that translational control might be important in coordinating the two genomes. Follow-up studies revealed that $1 \mu \mathrm{M}$ cycloheximide elevated nuOXPHOS 1.3-fold but decreased mtOXPHOS 2.4-fold (Fig. 2c). Interestingly, we found that nuOXPHOS expression, but not mtOXPHOS expression, correlated strongly with cellular ATP levels (Figure 2B). To determine whether the nuOXPHOS expression drives the changes in ATP levels, or reacts to changes in ATP levels, we performed follow-up, time-course analyses with $20 \mu \mathrm{M}$ perphenazine, a compound that dropped nuOXPHOS. We found that nuOXPHOS expression declined significantly $(21 \%$, t-test, $p=0.004)$ within the first hour of treatment, while cellular ATP levels remained unchanged $(0.6 \%$, t-test, $p=0.84)$ at these early time points. We determined that we were well powered to detect a 5\% change in ATP levels, supporting the notion that ATP levels were unchanged. At later time points, however, ATP levels did drop significantly ( $8 \mathrm{hrs:} 11 \%$ decrease, t-test, $p=1.4 \times 10^{-5}, 24 \mathrm{hrs:} 27 \%$ decrease, $\mathrm{t}$-test, $p=6.3 \times 10^{-22}$ ), suggesting that the decline in nuOXPHOS expression precedes and drives the decline in cellular ATP levels.

\section{Exploring the mitochondrial basis for drug toxicity}

Second, we were interested in applying our compendium to probe the role of mitochondria in human drug toxicity. We focused on the HMG-CoA reductase inhibitors (statins). Statins are taken by nearly 100 million patients worldwide and are associated with a $0.1-0.5 \%$ incidence of myopathy27. It is believed that the myopathy may be caused by a depletion of ubiquinone pools, which can result in blockade of the electron transport chain28. However, clinical and epidemiological studies have produced conflicting results on the association between statins and myopathy28. Of the six statins present in our screening collection, three gave strong decreases in cellular ATP levels and MTT activity (Fig. 3a). Previous studies have shown that lovastatin and simvastatin reduce MTT activity and ATP levels29, consistent with our screening results. To eliminate the possibility that we uncovered two classes based merely on potency, we measured cellular ATP levels over a range of doses up to $40 \mu \mathrm{M}$. We observed the same segregation of effects, with atorvastatin, pravastatin, and rosuvastatin showing little to no effect on cellular/mitochondrial ATP levels (Supplementary Fig. 3 online).

To determine whether this profile represents a signature of drug-induced myopathy, we established a centroid profile for the three mitochondria-active statins (fluvastatin, lovastatin, and simvastatin), and sought to identify other clinically used drugs with a similar assay profile. The nearest-neighbor drugs to the centroid statin profile (Fig. 3b) were amoxapine, cyclobenzaprine, propranolol, griseofulvin, pentamidine, paclitaxel, propafenone, ethaverine, trimeprazine, and amitriptyline. Remarkably, five of these compounds (amoxapine, propranolol, griseofulvin, pentamidine, and paclitaxel) have also 
been associated with skeletal muscle myopathy or myalgia30-34, whereas only a small fraction of all FDA-approved drugs are believed to be associated with this side effect35. This suggests that the drug profile might be indicative of myopathy. Further examination of the screening data revealed that two known electron transport chain inhibitors (betadihydrorotenone, a complex I inhibitor and antimycin A, a complex III inhibitor) were among the 16 nearest-neighbor compounds to this assay profile, which sheds mechanistic insight onto this profile. Together, the data support the idea that myopathy induced by these five other drugs could be mitochondrial in origin.

We were struck by the fact that one of these nearest neighbor compounds is propranolol, a widely used antihypertensive agent. Follow-up experiments confirmed that propranolol decreases cellular ATP levels in a dose-dependent manner, whereas other selective beta-1 blockers do not (Supplementary Fig. 3 online). Because many patients take both a statin and a beta-blocker for cardioprotection, we were curious to know if the two drugs might interact in toxicity. We thus assessed cellular ATP levels after treating with all possible combinations of the six statins in our collection and three beta-blockers (atenolol, metoprolol, and propranolol), with concentrations all falling between 2.5 and $10 \mu \mathrm{M}$ (Figure $3 c)$. While neither atenolol nor metoprolol displayed an effect either alone or in combination with any statin, propranolol had an additive effect on statin-induced decrease in ATP levels, using the Bliss independence model (Figure 3c)36. Importantly, our screening compendium and follow-up experiments (Figure 3c) raise the important clinical hypothesis that patients on a combination of propranolol and one of the three mitochondrially active statins might be at a higher risk for developing myopathy.

\section{Discovering lead compounds for common degenerative diseases}

Finally, we were interested in applying the screening compendium to search for lead compounds that might hold therapeutic potential for common human diseases. We and others have recently shown that a decline in OXPHOS gene expression and an elevation in ROS generation are associated with type- 2 diabetes $4,37,38$, neurodegeneration 39 , and the aging process itself5,6. We therefore queried our compendium to identify compounds that might be capable of elevating OXPHOS expression while reducing ROS generation.

We used two computational strategies to spotlight such compounds (see Methods). First, we sought to determine if any structurally related set of compounds might boost OXPHOS expression while also suppressing ROS levels, and developed a simple analytical strategy to answer this problem (Supplementary Table 3 online). This strategy involves organizing all compounds based on structural similarity (see Methods), and then asking whether members of a cluster exhibit concordant scores in a given assay (Fig. 4a). The advantage of this strategy is that individual compounds might exhibit a subtle response, not detectable in a primary screen with duplicate measurements, whereas the grouped analysis provides added statistical power. Second, in a complementary approach, we sought to identify individual compounds that promote OXPHOS gene expression while reducing ROS levels. The advantage of this method is that it can reveal structurally unrelated compounds that individually exert large effect sizes in the two assays of interest. We focused on the upper 
tail of the combined nuclear and mitochondrial OXPHOS gene expression distribution (Fig. $4 b)$.

Remarkably, both analytical strategies spotlighted microtubule modulators, including both a microtubule stabilizer (paclitaxel) and several destabilizers (mebendazole, nocodazole, podophyllotoxin, and vinblastine), as agents that boost OXPHOS expression while suppressing ROS levels. The second strategy also yielded deoxysappanone B, a natural product found in sappan wood40, whose molecular mode of action is unknown and has not been previously linked to microtubule biology. The other microtubule inhibitors within the compound collection (colchicine and griseofulvin) did not display the same decrease in ROS levels, but did show a modest increase in OXPHOS expression.

Next, we were interested in confirming these primary screening results and determining whether the effects on OXPHOS expression and ROS levels occur via shared or distinct mechanisms, and whether these were on-target or off-target effects of microtubule disruption. We therefore re-tested the microtubule modulators at a range of $20 \mathrm{nM}$ to $20 \mu \mathrm{M}$ (Fig. 5a). Treatment with either deoxysappanone B, mebendazole, nocodazole, podophyllotoxin, or vinblastine increased OXPHOS expression and decreased ROS levels at the same dose of $2 \mu \mathrm{M}$, while paclitaxel showed effects in the two assays at $20 \mathrm{nM}$, suggesting a shared mechanism for OXPHOS expression and ROS level. Importantly, at these doses, these compounds did not decrease cell viability (Fig. 5a), indicating that the decline in ROS is not simply a reflection of overt cytotoxicity. We also imaged tubulin immunofluorescence after treatment with deoxysappanone B and paclitaxel, two compounds that showed distinct potencies. For both compounds, the potency required for microtubule disruption was the same as that required to affect OXPHOS expression and ROS level (Supplementary Fig. 4 online). To our knowledge, deoxysappanone B has not previously been linked to microtubule inhibition, but now has been predicted to do so and validated by this study. Given that structurally and mechanistically diverse microtubule modulators increased OXPHOS gene expression, decreased cellular ROS, and disrupted microtubules with equivalent potencies, it is likely that these effects are directly related to inhibition of microtubules, and not due to an off-target effect.

Because mtDNA replication and transcription are often coupled41, we sought to determine whether any of these compounds promoted mtDNA replication. Several of these microtubule modulators increased mtDNA copy number by approximately 3 -fold (Fig. 5b), while podophyllotoxin and vinblastine were unable to increase mtDNA copy number at the concentrations tested.

We sought to determine the transcriptional mechanism by which microtubule inhibition might promote OXPHOS expression and mtDNA replication while suppressing ROS. We hypothesized that these changes might be occurring via PGC-1a, a transcriptional coactivator that regulates mitochondrial biogenesis in muscle42, and whose transcriptional program is diminished in type- 2 diabetes 4 . Consistent with this hypothesis, both mebendazole and deoxysappanone B induced PGC-1a gene expression by approximately 3fold (Fig. 5c). We have previously shown that the transcription factor ERRa serves as a key transcriptional partner of PGC-1a to drive OXPHOS expression in muscle, and that 
disruption of ERRa with the selective inverse agonist XCT790 suppresses PGC-1a-induced OXPHOS expression43. Therefore, we tested whether XCT790 is capable of inhibiting compound-induced transcription. We observed that both mebendazole and deoxysappanone B increased the expression of a nuclear OXPHOS gene, Atp5a1, by approximately 20\%, and this increase was completely inhibited by XCT790 (Fig. 5d), further suggesting a PGC-1adependent mechanism of compound activity. The mitochondrial ROS scavenger MnSOD is part of the same PGC-1a/ERRa pathway44, and since we observed decreased cellular ROS levels after treatment with these small molecules, we tested compound effects on this gene as well. A similar increase in MnSOD levels, which was suppressible by XCT790, was observed with these compounds (Fig. 5e). These results suggest that microtubule modulators both activate OXPHOS transcription and reduce cellular ROS levels in a PGC-1a/ERRadependent manner.

\section{DISCUSSION}

The mitochondrion is an extremely complex organelle, with components derived from both the nuclear and mitochondrial genomes, whose activity must be carefully coupled to cellular metabolism and signalling. We systematically investigated mitochondrial function using a chemical perturbation approach that involved multiple physiological and multiplexed gene expression assays for OXPHOS. The gene expression and physiological assays provided complementary information, and were jointly useful in interpreting the effects of compound treatment. The compendium is freely available and can be used to investigate the network properties of the mitochondrion. Moreover, because so many of the 2490 compounds tested are well-characterized, they can be used immediately as tool compounds for studying mitochondrial biology. In the current study, we have already applied it to make three novel discoveries.

First, we focused on how the nuclear genome and mtDNA are coordinated across a variety of physiological states. We were able to pursue this question since our multiplexed gene expression assays interrogate both nuOXPHOS and mtOXPHOS transcripts. This is the first expression compendium to interrogate the expression of both nuclear and mitochondrial genomes. We found that the bulk of compounds coordinately regulated the expression from both genomes. However, we found that eukaryotic protein synthesis inhibitors disrupt the crosstalk between these two genomes. A previous study has shown that the calcium ionophore A-23187 can also elevate nuOXPHOS while decreasing mtOXPHOS45. We now have an array of tool compounds (Fig. 2) to investigate whether protein synthesis inhibitors also disrupt the nuclear-to-mitochondrial genome crosstalk via previously identified pathways or through a novel mechanism.

Second, we mined the compendium to learn about drug toxicity. We focused on statins, which are taken by nearly 100 million patients around the world, and have a $0.1-0.5 \%$ incidence of myopathy27. We observed a bimodal response from the HMG-CoA reductase inhibitors (Figure 3), with three compounds (atorvastatin, pravastatin, rosuvastatin) forming a group with no activity in our physiological assays, and three other compounds (fluvastatin, lovastatin, simvastatin) forming a group showing signs of OXPHOS inhibition. Statins block the synthesis of cholesterol, which is a precursor to ubiquinone, a mobile electron carrier in 
the mitochondrion that is critical to OXPHOS function. It has therefore been previously suggested that statin myopathy may be due to a respiratory chain blockade28. Our data suggest that these statins (fluvastatin, lovastatin, simvastatin) should be examined further for their effects on skeletal muscle ubiquinone and drug-induced myopathy. Using the statin toxicity signature (Fig. 3a), we also discovered that a widely used antihypertensive agent, propranolol, shares the same profile of mitochondrial inhibition. Beta-1-selective blockers, such as atenolol and metoprolol, however, did not exhibit this pattern of toxicity (Supplementary Figure 3 online). Our follow-up experiments revealed an additive interaction between the statins and propranolol on mitochondrial inhibition, which raises the distinct hypothesis that patients taking both statins and propranolol might be at increased risk for developing skeletal muscle myopathy. Because many patients with heart disease are likely to be on this drug combination, this hypothesis can be tested easily and may help account for the conflicting reports on skeletal muscle myopathy associated with statins.

Third, we queried our compendium for compounds that could potentially reverse signatures associated with age-associated, degenerative diseases. We and others have recently reported that a decline in OXPHOS expression and a rise in ROS generation accompany a number of common degenerative diseases, including diabetes, neurodegeneration, and the aging process itself4. Using two computational approaches, we discovered that structurally unrelated microtubule modulators with diverse mechanisms of action (both stabilizers and destabilizers) increased OXPHOS expression and decreased cellular ROS. Follow-up dose response studies of mitochondrial function and microtubule disruption suggest that these drugs are affecting OXPHOS expression and ROS levels via an on-target mechanism.

Our studies raise the possibility that manipulation of the microtubule pathway may reverse the gene expression and ROS signatures associated with common degenerative diseases and that these may represent therapeutic targets. Previous clinical case reports support this hypothesis. Type 1- and type-2 diabetics treated for parasitic worm infections with mebendazole unexpectedly showed improvement both in fasting blood glucose levels and non-esterified fatty acid metabolism46. Blood glucose levels improved without weight loss, suggesting that the improvement in diabetes was not due to a toxic side effect of mebendazole. Moreover, deoxysappanone B, a natural product found in sappan wood, has been reported to be an anti-diabetic component of Chinese herbal medicine, though the mechanism was unclear40. Our data convincingly demonstrate that deoxysappanone B is a microtubule disruptor (Supplementary Fig. 4 online).

At a molecular level, we have uncovered an unexpected link between microtubule disruption and an increase in PGC-1a/ERRa-mediated OXPHOS gene expression that may be relevant for understanding disease pathogenesis. Previous studies have demonstrated changes in mitochondrial stability and morphology after treatment with microtubule inhibitors47, although none have specifically documented their effects on OXPHOS expression and ROS levels. It is intriguing to speculate that perhaps interactions between the cytoskeleton and the mitochondrion are important in integrating cellular homeostasis across the cell cycle. Of note, many of these microtubule modulators are used for treating cancer; our results may therefore have implications for understanding the metabolic basis for chemotherapeutic action. 
While decades of research in bioenergetics have yielded a detailed, mechanistic understanding of OXPHOS in isolated mitochondria48, our screening compendium provides a foundation for understanding how OXPHOS physiology and regulation are integrated within the broader signaling and metabolic network of the cell. Each small molecule probe modulates a different aspect of cell biology, and our compendium provides insights into how OXPHOS remodels at the level of physiology and gene expression. Because many of the small molecules utilized in this study are well-characterized bioactives, our compendium provides a rich chemical toolkit for manipulating mitochondria in cells and in animals in a defined manner. Given the growing number of rare and common diseases associated with different types of mitochondrial dysfunction, we anticipate that our compendium will serve as a generic tool for systematically investigating mitochondrial pathogenesis and for restoring mitochondrial function.

\section{METHODS}

\section{Cell culture}

C2C12 myoblasts (ATCC; Manassas, VA) were grown in Dulbecco's Modified Eagle's Medium (DMEM, Mediatech; Herndon, VA) supplemented with 10\% fetal bovine serum and antibiotics $(100 \mu \mathrm{g} / \mathrm{mL}$ penicillin/streptomycin mix) in a humidified atmosphere at 37 ${ }^{\circ} \mathrm{C}$ with $5 \% \mathrm{CO}_{2}$. Differentiation into myotubes was induced at $80 \%$ density on "day 0" by changing media to DMEM supplemented with $2 \%$ horse serum.

\section{Cell-based high-throughput screening}

For all screening, 4,000 C2C12 myoblasts were seeded per well of either black or white 384well optical bottom plates (Nunc; Rochester, NY) at $50 \mu \mathrm{L} /$ well. On day 4 of differentiation, $100 \mathrm{~nL}$ compound was pin-transferred in duplicate into fresh media with a steel pin array, using the CyBi-Well robot (CyBio; Woburn, MA). In order to increase the number of mocktreated wells included in the control distribution, we added an additional plate containing DMSO alone. Compound-treated plates were incubated at $37{ }^{\circ} \mathrm{C}$ for 48 hours. All cell-based assay measurements were performed using the EnVision plate reader (PerkinElmer; Waltham, MA). The coefficient of variation for each of these assays was estimated to be less than 15\%. All data has been deposited in ChemBank: http://chembank.broad.harvard.edu/ assays/view-project.htm?id=1000453.

Calcein viability assay-Media was aspirated from plates, and $30 \mu \mathrm{L} /$ well $1 \mu \mathrm{M}$ calceinAM (Molecular Probes; Carlsbad, CA) in phenol red-free media was added. Plates were incubated for 1 hour at $37{ }^{\circ} \mathrm{C}$ and washed three times with $50 \mu \mathrm{L} /$ well PBS. Fluorescence was measured at ex/em $485 \mathrm{~nm} / 530 \mathrm{~nm}$.

JC-1 mitochondrial membrane potential assay-Upon depolarization, the dye is converted from a diffuse green form to red fluorescent J-aggregates. The ratio of red to green fluorescence serves as a readout of the mitochondrial membrane potential. Media was aspirated from plates, and $20 \mu \mathrm{L} /$ well $3.25 \mu \mathrm{M} \mathrm{JC}-1$ (Molecular Probes) in phenol red-free media was added. Plates were incubated for 2 hours at $37{ }^{\circ} \mathrm{C}$ and washed three times with 50 
$\mu \mathrm{L} /$ well PBS. Fluorescence was measured, first at ex/em $530 \mathrm{~nm} / 580 \mathrm{~nm}$ ("red"), followed by ex/em $485 \mathrm{~nm} / 530 \mathrm{~nm}$ ("green”).

Assay for cellular ATP levels-20 $\mu \mathrm{L} /$ well CellTiterGlo reagent (Promega; Madison, WI) was added to $20 \mu \mathrm{L} /$ well cell-culture media. Plates were agitated for 2 minutes and incubated for 10 minutes at room temperature before measuring luminescence.

MTT assay-Media was aspirated from plates, and $50 \mu \mathrm{L} / \mathrm{well} 0.5 \mathrm{mg} / \mathrm{mL}$ MTT (3-(4, 5dimethylthiazol-2-yl)-2,5-diphenyltetrazolium bromide, Calbiochem; San Diego, CA) in phenol red-free media was added. Plates were incubated for 2 hours at $37^{\circ} \mathrm{C}$, followed by aspiration of MTT solution, addition of $50 \mu \mathrm{L} /$ well DMSO to dissolve formazan crystals, and incubation at $37{ }^{\circ} \mathrm{C}$ for 30 minutes. After incubation, plates were equilibrated to room temperature for an additional 20-30 minutes. Absorbance was measured at $540 \mathrm{~nm}$.

Reactive oxygen species assay-Media was aspirated from plates, and $20 \mu \mathrm{L} /$ well 10 $\mu \mathrm{M}$ CM-H2DCFDA (5-(and-6)-chloromethyl-2', $7^{\prime}$-dichlorodihydrofluorescein diacetate, Molecular Probes) in phenol red-free media was added. Plates were incubated for 1 hour at $37{ }^{\circ} \mathrm{C}$ and washed three times with $50 \mu \mathrm{l} /$ well PBS. Fluorescence was measured at ex/em $485 \mathrm{~nm} / 530 \mathrm{~nm}$.

Cytochrome c protein detection-Cells were fixed with $3.7 \%$ formaldehyde in PBS for 30 minutes, followed by washing with TBS containing 0.1\% Tween-20 (TBST) and blocking with TBST $+3 \%$ BSA for 1 hour at room temperature. Cytochrome $c$ was detected by incubating with primary antibody (Cell Signaling Technology, Danvers, MA; 1:100) overnight at $4{ }^{\circ} \mathrm{C}$, washing three times with TBST, and incubating with secondary antibody (Alexa Fluor 488-conjugated anti-mouse IgG, Invitrogen; 1:250) for 1 hour at room temperature. Plates were washed three times with TBST and fluorescence measured at ex/em $485 \mathrm{~nm} / 530 \mathrm{~nm}$.

\section{Gene expression-based high-throughput screening}

To narrow down the list of potential genes from nearly 100 nuclear-encoded OXPHOS genes, we used a list of highly co-regulated set of OXPHOS genes4 that are coordinately expressed across tissues and are downstream of the PGC-1a transcriptional coactivator. From this list, we selected genes that showed the highest signal-to-noise ratio in the microarray analysis of PGC-1a over-expression in $\mathrm{C} 2 \mathrm{C} 12$ myotubes 43 representing all five OXPHOS complexes. We also selected two genes that are downregulated by PGC-1a with the best signal-to-noise ratio. As controls, we selected genes that showed the lowest signal (no treatment effect) and lowest noise (biological variation) in the PGC-1a over-expression data43, as well as genes previously found to be invariant from the analysis of multiple microarray datasets 49 . We selected control genes that span a wide range of expression levels to prevent biasing for abundant transcripts. The selected OXPHOS transcripts capture the bulk of the variation exhibited by the OXPHOS transcripts represented on over 5000 publicly available mouse microarrays on the Affymetrix platform (data not shown).

From the list of OXPHOS genes and control genes for GE-HTS, we designed probe pairs with T7 and T3 universal primer sites, 40-bp target sequence split into two 20-bp sequences 
for each probe, and gene-specific barcode sequence attached to the 5' probe according to the published assay specification19. We selected 40-bp gene-specific target sequences that are not alternatively spliced using oligonucleotide sequences found in the Mouse Exonic Evidence-Based Oligonucleotide Chip (MEEBO, http://alizadehlab.stanford.edu/). Full primer sequences are included in Supplementary Table 4 online.

The GE-HTS assay was performed as previously described50. Because this assay measures the final amount of PCR products rather than the real-time measurement of gene expression, we adjusted the parameters in the original protocol so that our genes were within the linear range of the assay. We removed $20 \mu \mathrm{L}$ of media and added $25 \mu \mathrm{L}$ of lysis buffer in 384-well, and used 24 PCR cycles instead of the 29 cycles described50. We used 32 DMSO-treated and 32 PGC-1a adenovirus-treated wells per 384-well compound plate, with one additional control plate containing 192 DMSO-treated wells, 32 GFP adenovirus-treated wells, and 160 PGC-1a adenovirus-treated wells.

\section{Tubulin immunofluorescence}

On day 4 of differentiation, $\mathrm{C} 2 \mathrm{C} 12$ myotubes were treated with compound for 48 hours, and then fixed for 5 minutes in ice-cold $100 \%$ methanol. Cells were washed once in $50 \mu \mathrm{L}$ PBSTB2 (PBS with 0.1\% Tween-20 and 2\% BSA) and blocked in PBSTB2 for 1 hour at room temperature or overnight at $4{ }^{\circ} \mathrm{C}$. Cells were incubated with an anti-a-tubulin (SigmaAldrich; St. Louis, MO) antibody, 1:1,000 in PBSTB2, for 1 hour at room temperature, followed by three washes with PBSTB2. Cells were incubated with secondary antibody (Alexa 488-conjugated anti-mouse antibody, 1:500 in PBSTB2) (Molecular Probes) and Hoechst 33342 for 1 hour at room temperature, followed by three washes in PBSTB2. Cells were visualized using an automated microscope (IX-Micro, Molecular Devices, Sunnyvale, CA).

\section{Quantitative PCR of mtDNA and transcripts}

mtDNA quantification-Mitochondrial DNA copy numbers were assessed by quantifying the relative amount of mitochondrial-encoded Co1 copy number and the nuclear-encoded $\beta$ actin copy number. DNA from cells were extracted using DNeasy (Qiagen; Valencia, CA) and quantified for Co1 and $\beta$-actin copy number using quantitative PCR (Applied Biosystems; Foster City, CA). The change in the Co1 to $\beta$-actin ratio between the compound-treated and DMSO control represents the fold change in mtDNA copy number.

Gene expression-We extracted RNA using an RNeasy kit (Qiagen) and synthesized cDNA using a high-capacity cDNA reverse transcription kit (Applied Biosystems) with random hexamers, as described by the manufacturer. The cDNA was then used for real-time PCR quantitation of products for mouse Atp5a1 (Mm00431960_m1), MnSOD (Mm01313000_m1), and Ppargc1a (Mm00447183_m1), with Hprt1 (Mm03024075_m1) serving as an internal control, using TaqMan gene-expression assays (Applied Biosystems). 


\section{Statistics}

Cell-based screening-Composite $Z$-scores reflecting compound performance as compared to a mock-treated (DMSO) distribution were calculated as described20,21 (see also http://chembank.broad.harvard.edu/details.htm?tag=Help\#screeningData).

GE-HTS-We first eliminated wells that failed the assay reaction by filtering out wells in which the raw expression value of Rps2 (a control gene) was two standard deviations below the median DMSO control value for each plate. We normalized for plate-to-plate variation by scaling the per-well expression level of each gene to the median expression level of that gene in PGC-1a control wells on each plate. We set the median PGC-1a expression value for each gene to 1 , and then normalized for well-to-well variation by dividing the expression level of each OXPHOS gene by the average value of eight control genes for each well. This number represents the processed data value.

To score the expression level of 12 nuclear- and 13 mitochondria- encoded OXPHOS genes, we first weighted each gene by its ability to distinguish DMSO control wells from PGC-1atreated wells. We calculated the signal-to-noise ratio49 of each gene using our PGC-1a positive control and DMSO negative control, and multiplied the expression value of each gene per well by this signal-to-noise ratio. We then summed these weighted scores over nuclear-encoded or mitochondrial-encoded OXPHOS genes to derive one score each for expression within each genome. Composite $Z$-scores were calculated as described above.

Similarity between assay profiles-We used the cell-based composite $Z$-scores from the ATP, MTT, JC1, and ROS assays to calculate the root-mean-square distance between performance vectors, since this statistic gives greater weight to values far from zero. Centroid statin scores were achieved by taking the arithmetic mean of the composite $Z$ scores from these four assays.

Chemical set analysis-We used Pipeline Pilot (Scitegic; San Diego, CA) to perform $K$ means clustering of the molecules based on common and biologically intuitive chemical features (molecular weight, octanol-water partition coefficient, number of hydrogen bond donors and acceptors, and number of rotatable bonds). We set $K$ to 624 to result in an average of 5 compounds per cluster. In order to detect enrichment for assay performance within each compound cluster, we performed the Mann-Whitney rank sum test on each cluster in each assay.

\section{Supplementary Material}

Refer to Web version on PubMed Central for supplementary material.

\section{Acknowledgements}

\footnotetext{
We thank Stephanie Norton, Jason Burbank, Mariah Eustice, and Nicky Tolliday for assistance in high-throughput screening; Nathan Billings and Olga Goldberger for technical assistance; Oded Shaham, Ken Ross, and Paul Clemons for computational assistance; and Alice Ting, Eric Lander, and Robert Gould for thoughtful discussions and comments on the manuscript. S.L.S. and T.R.G. are Investigators of the Howard Hughes Medical Institute. V.K.M. is recipient of a Career Award in the Biomedical Sciences from the Burroughs Wellcome Fund, a Charles E. Culpeper Scholarship in Medical Science, and a Physician Scientist Early Career Award from the Howard
} 
Hughes Medical Institute. This work was supported by grants from the National Institute of Health (NIDDK), the American Diabetes Association, and the Richard and Susan Smith Family Foundation (V.K.M.).

\section{References}

1. Anderson S, et al. Sequence and organization of the human mitochondrial genome. Nature. 1981; 290:457-465. [PubMed: 7219534]

2. Chance B, Williams GR. Respiratory enzymes in oxidative phosphorylation. III. The steady state. J. Biol. Chem. 1955; 217:409-427. [PubMed: 13271404]

3. DiMauro S, Schon EA. Mitochondrial respiratory-chain diseases. N. Engl. J. Med. 2003; 348:26562668. [PubMed: 12826641]

4. Mootha VK, et al. PGC-1alpha-responsive genes involved in oxidative phosphorylation are coordinately downregulated in human diabetes. Nat. Genet. 2003; 34:267-273. [PubMed: 12808457]

5. Petersen KF, et al. Mitochondrial dysfunction in the elderly: possible role in insulin resistance. Science. 2003; 300:1140-1142. [PubMed: 12750520]

6. Balaban RS, Nemoto S, Finkel T. Mitochondria, oxidants, and aging. Cell. 2005; 120:483-495. [PubMed: 15734681]

7. Kelly DP, Scarpulla RC. Transcriptional regulatory circuits controlling mitochondrial biogenesis and function. Genes Dev. 2004; 18:357-368. [PubMed: 15004004]

8. Weinstein JN, et al. An information-intensive approach to the molecular pharmacology of cancer. Science. 1997; 275:343-349. [PubMed: 8994024]

9. Hughes TR, et al. Functional discovery via a compendium of expression profiles. Cell. 2000; 102:109-126. [PubMed: 10929718]

10. Lamb J, et al. The Connectivity Map: using gene-expression signatures to connect small molecules, genes, and disease. Science. 2006; 313:1929-1935. [PubMed: 17008526]

11. Ramanathan A, Wang C, Schreiber SL. Perturbational profiling of a cell-line model of tumorigenesis by using metabolic measurements. Proc. Natl. Acad. Sci. USA. 2005; 102:59925997. [PubMed: 15840712]

12. Leary SC, Battersby BJ, Hansford RG, Moyes CD. Interactions between bioenergetics and mitochondrial biogenesis. Biochim Biophys Acta. 1998; 1365:522-530. [PubMed: 9711303]

13. Dolma S, Lessnick SL, Hahn WC, Stockwell BR. Identification of genotype-selective antitumor agents using synthetic lethal chemical screening in engineered human tumor cells. Cancer Cell. 2003; 3:285-296. [PubMed: 12676586]

14. Smiley ST, et al. Intracellular heterogeneity in mitochondrial membrane potentials revealed by a Jaggregate-forming lipophilic cation JC-1. Proc. Natl. Acad. Sci. USA. 1991; 88:3671-3675. [PubMed: 2023917]

15. Berridge MV, Tan AS. Characterization of the cellular reduction of 3-(4,5-dimethylthiazol-2yl)-2,5-diphenyltetrazolium bromide (MTT): subcellular localization, substrate dependence, and involvement of mitochondrial electron transport in MTT reduction. Arch. Biochem. Biophys. 1993; 303:474-482. [PubMed: 8390225]

16. Crouch SP, Kozlowski R, Slater KJ, Fletcher J. The use of ATP bioluminescence as a measure of cell proliferation and cytotoxicity. J. Immunol. Methods. 1993; 160:81-88. [PubMed: 7680699]

17. Ye G, Metreveli NS, Ren J, Epstein PN. Metallothionein prevents diabetes-induced deficits in cardiomyocytes by inhibiting reactive oxygen species production. Diabetes. 2003; 52:777-783. [PubMed: 12606520]

18. Stegmaier K, et al. Gene expression-based high-throughput screening (GE-HTS) and application to leukemia differentiation. Nature Genet. 2004; 36:257-263. [PubMed: 14770183]

19. Peck D, et al. A method for high-throughput gene expression signature analysis. Genome Biol. 2006; 7:R61. [PubMed: 16859521]

20. Kim YK, et al. Relationship of stereochemical and skeletal diversity of small molecules to cellular measurement space. J. Am. Chem. Soc. 2004; 126:14740-14745. [PubMed: 15535697]

21. Franz AK, Dreyfuss PD, Schreiber SL. Synthesis and cellular profiling of diverse organosilicon small molecules. J. Am. Chem. Soc. 2007; 129:1020-1021. [PubMed: 17263369] 
22. Larsson NG, Clayton DA. Molecular genetic aspects of human mitochondrial disorders. Annu. Rev. Genet. 1995; 29:151-178. [PubMed: 8825472]

23. Clayton DA. Transcription of the mammalian mitochondrial genome. Annu. Rev. Biochem. 1984; 53:573-594. [PubMed: 6383200]

24. Antonetti DA, Reynet C, Kahn CR. Increased expression of mitochondrial-encoded genes in skeletal muscle of humans with diabetes mellitus. J. Clin. Invest. 1995; 95:1383-1388. [PubMed: 7533791]

25. Huang $X$, et al. Insulin-regulated mitochondrial gene expression is associated with glucose flux in human skeletal muscle. Diabetes. 1999; 48:1508-1514. [PubMed: 10426366]

26. Heddi A, Stepien G, Benke PJ, Wallace DC. Coordinate induction of energy gene expression in tissues of mitochondrial disease patients. J. Biol. Chem. 1999; 274:22968-22976. [PubMed: 10438462]

27. Graham DJ, et al. Incidence of hospitalized rhabdomyolysis in patients treated with lipid-lowering drugs. JAMA. 2004; 292:2585-2590. [PubMed: 15572716]

28. Dirks AJ, Jones KM. Statin-induced apoptosis and skeletal myopathy. Am. J. Physiol. 2006; 291:C1208-C1212.

29. van Vliet AK, Negre-Aminou P, van Thiel GC, Bolhuis PA, Cohen LH. Action of lovastatin, simvastatin, and pravastatin on sterol synthesis and their antiproliferative effect in cultured myoblasts from human striated muscle. Biochem. Pharmacol. 1996; 52:1387-1392. [PubMed: 8937448]

30. Frendin TJ, Swainson CP. Acute renal failure secondary to non-traumatic rhabdomyolysis following amoxapine overdose. N. Z. Med. J. 1985; 98:690-691. [PubMed: 3863031]

31. Blessing W, Walsh JC. Myotonia precipitated by propranolol therapy. Lancet. 1977; 1:73-74. [PubMed: 63714]

32. Davidson BK. Myositis associated with griseofulvin therapy. Am. Fam. Physician. 1995; 52:1277. [PubMed: 7572547]

33. Delobel P, Pradinaud R. Rhabdomyolysis associated with pentamidine isethionate therapy for American cutaneous leishmaniasis. J. Antimicrob. Chemother. 2003; 51:1319-1320. [PubMed: 12668576]

34. Rowinsky EK, et al. Phase I and pharmacologic study of paclitaxel and cisplatin with granulocyte colony-stimulating factor: Neuromuscular toxicity is dose-limiting. J. Clin. Oncol. 1993; 11:20102020. [PubMed: 7692001]

35. Aronson, JK., editor. Meyler's Side Effects of Drugs. 15th edition.. Burlington, Massachusetts, USA: Elsevier Science; 2006.

36. Bliss CI. The toxicity of poisons applied jointly. Ann Appl Biol. 1939; 26:585-615.

37. Kelley DE, He J, Menshikova EV, Ritov VB. Dysfunction of mitochondria in human skeletal muscle in type 2 diabetes. Diabetes. 2002; 51:2944-2950. [PubMed: 12351431]

38. Houstis N, Rosen ED, Lander ES. Reactive oxygen species have a causal role in multiple forms of insulin resistance. Nature. 2006; 440:944-948. [PubMed: 16612386]

39. Lustbader JW, et al. ABAD directly links Abeta to mitochondrial toxicity in Alzheimer's disease. Science. 2004; 304:448-452. [PubMed: 15087549]

40. Li WL, Zheng HC, Bukuru J, De Kimpe N. Natural medicines used in the traditional Chinese medical system for therapy of diabetes mellitus. J. Ethnopharmacol. 2004; 92:1-21. [PubMed: 15099842]

41. Bonawitz ND, Clayton DA, Shadel GS. Initiation and beyond: multiple functions of the human mitochondrial transcription machinery. Mol. Cell. 2006; 24:813-825. [PubMed: 17189185]

42. Wu Z, et al. Mechanisms controlling mitochondrial biogenesis and respiration through the thermogenic coactivator PGC-1. Cell. 1999; 98:115-124. [PubMed: 10412986]

43. Mootha VK, et al. Erralpha and Gabpa/b specify PGC-1alpha-dependent oxidative phosphorylation gene expression that is altered in diabetic muscle. Proc. Natl. Acad. Sci. USA. 2004; 101:65706575. [PubMed: 15100410] 
44. Valle I, Alvarez-Barrientos A, Arza E, Lamas S, Monsalve M. PGC-1alpha regulates the mitochondrial antioxidant defense system in vascular endothelial cells. Cardiovasc. Res. 2005; 66:562-573. [PubMed: 15914121]

45. Freyssenet D, Irrcher I, Connor MK, DiCarlo M, Hood DA. Calcium-regulated changes in mitochondrial phenotype in skeletal muscle cells. Am. J. Phys. 2004; 286:1053-1061.

46. Caprio S, et al. Improvement of metabolic control in diabetic patients during mebendazole administration: preliminary studies. Diabetologia. 1984; 27:52-55. [PubMed: 6381196]

47. Lee CF, Liu CY, Hsieh RH, Wei YH. Oxidative stress-induced depolymerization of microtubules and alteration of mitochondrial mass in human cells. Ann. N. Y. Acad. Sci. 2005; 1042:246-254. [PubMed: 15965069]

48. Mitchell P. Coupling of phosphorylation to electron and hydrogen transfer by a chemi-osmotic type of mechanism. Nature. 1961; 199:144-148. [PubMed: 13771349]

49. Lee PD, Sladek R, Greenwood CM, Hudson TJ. Control genes and variability: absence of ubiquitous reference transcripts in diverse mammalian expression studies. Genome Res. 2002; 12:292-297. [PubMed: 11827948]

50. Hieronymus $\mathrm{H}$, et al. Gene expression signature-based chemical genomic prediction identifies a novel class of HSP90 pathway modulators. Cancer Cell. 2006; 10:321-330. [PubMed: 17010675] 


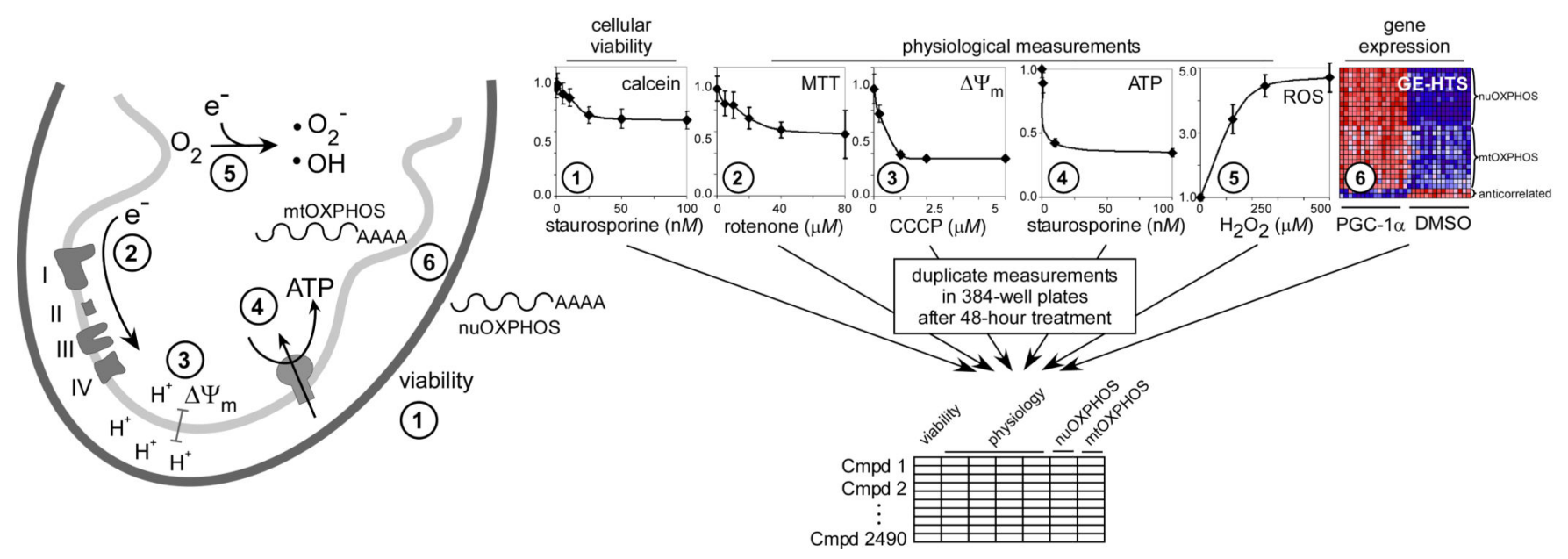

Figure 1. Complementary profiles of mitochondrial physiology and mitochondrial gene expression across 2490 chemical perturbations Multiple assays were used to monitor OXPHOS function and regulation over a large collection of chemical perturbations. Calcein (1) measures cell viability and is used to filter out compounds with obvious toxicity effects, with the protein kinase inhibitor staurosporine used as a positive control. The MTT assay (2) measures cellular dehydrogenase activity, primarily from the electron transport chain; rotenone, a complex I inhibitor, was used to decrease MTT activity. The JC-1 assay (3) measures the mitochondrial membrane potential $\left(\Delta \Psi_{\mathrm{m}}\right)$, and is inhibited acutely ( 1 hour) by the addition of the uncoupler CCCP. A commercially available kit is used to measure ATP (4) levels through luciferase activity; staurosporine drops ATP levels in a dose-dependent manner. CM-H2DCFDA measures cellular ROS levels (5), which are generated primarily in the mitochondria. Hydrogen peroxide increases this measurement in a dose-dependent manner. The expression of both nuclear and mitochondrial OXPHOS genes is measured by a multiplex PCR technique called GE-HTS (6). Each column represents one sample from a 384-well plate; expression levels for each gene are represented as a row-normalized heat map. The assay can readily distinguish the expression of control, DMSO-treated cells versus those cells expressing PGC-1a, an inducer of mitochondrial biogenesis. Each assay was performed in biological duplicate following 48 hour treatment with each of 2490 small molecules. The data from both physiological and gene expression-based assays were incorporated into the screening compendium. 
10
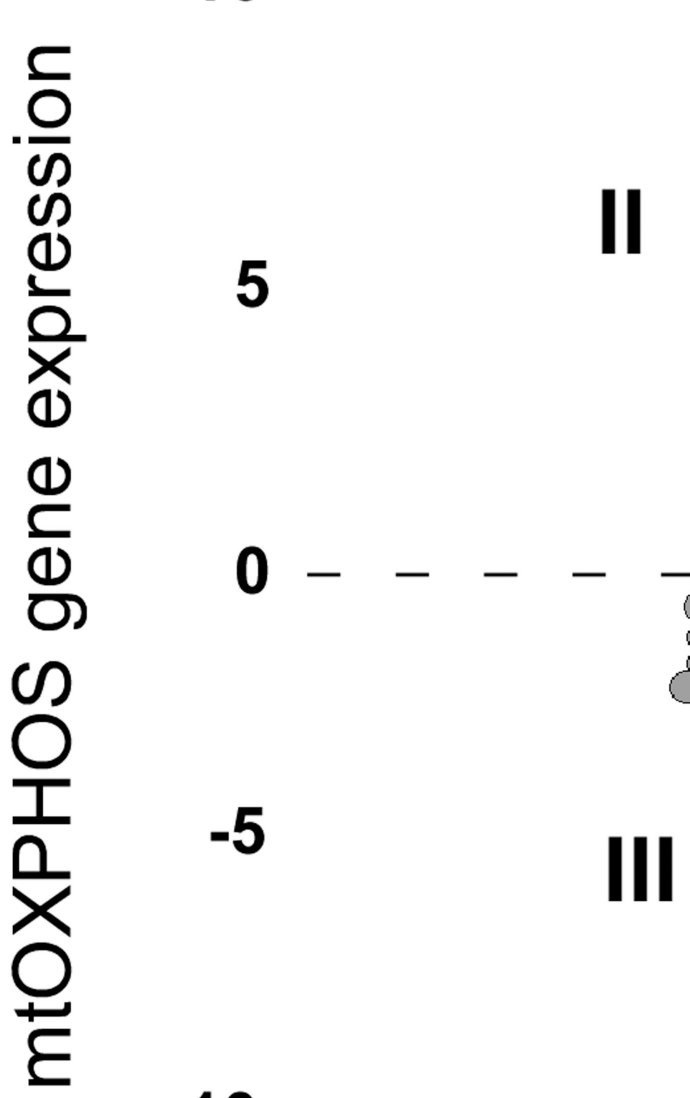

$-10$

$-10$

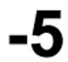

0

5

\section{nuOXPHOS gene expression}

Figure 2. Coupling of nuclear and mitochondrial OXPHOS expression

(a) The GE-HTS assay design allowed us to separate gene-expression scores into those specific for nuclear- and mitochondrial-encoded OXPHOS genes (nuOXPHOS and mtOXPHOS, respectively). A two-dimensional plot of the composite Z-scores for nuOXPHOS and mtOXPHOS expression is shown. (b) Row-normalized heat map displaying the top fifteen compounds in each quadrant, with the same numbering indicated in ' $a$ '. nuOXPHOS and mtOXPHOS transcripts are shown above a heat map indicating cellular ATP levels. (c) Real-time PCR validation of select compounds at the indicated doses, using Atp5a1 (nuOXPHOS) and mt-CoI (mtOXPHOS) normalized to Hprt (internal control). Values indicate average fold change from mock-treated (DMSO) wells \pm standard deviation in four biological replicates. 


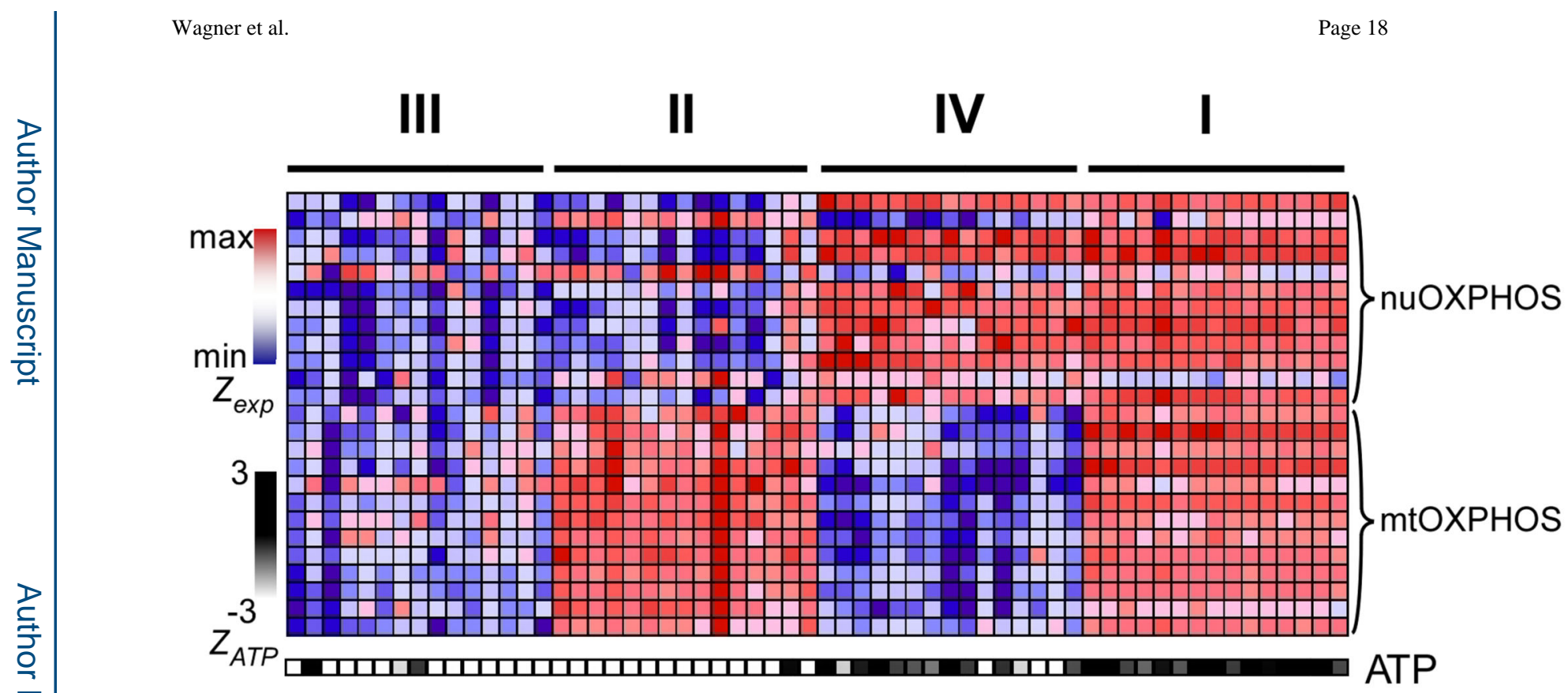

Figure 3. Statin induced mitochondrial toxicity

(a) Six of the HMG-CoA reductase inhibitors (statins) in clinical use are in the chemical screening collection. Composite Z-scores for cell viability, ATP generation, MTT activity, $\Delta \Psi_{\mathrm{m}}$, ROS generation, and gene expression are shown, where negative scores indicate a decrease in signal compared to mock-treated (DMSO) wells. The gray shading indicates scores that fall within the noise envelope. (b) A centroid statin score was generated by calculating the arithmetic means of the composite $Z$-scores for the class of compounds comprised of fluvastatin, lovastatin, and simvastatin. The ten nearest-neighbor clinically used drugs (amoxapine, cyclobenzaprine, propranolol, griseofulvin, pentamidine, paclitaxel, propafenone, ethaverine, trimeprazine, and amitriptyline) were identified by calculating the root-mean-square distance of each performance vector to the profile of interest. (c) All six statins in our collection were tested in combination with three clinically used beta adrenergic blockers for their effects on cellular ATP levels in $\mathrm{C} 2 \mathrm{C} 12$ myotubes. Compound concentrations are indicated on each axis of the heatmap, and the color indicates the change in ATP levels (ranging from black, for no change in cellular ATP levels, to yellow, for a $50 \%$ decrease in ATP levels). Data represent the average of six independent wells; coefficients of variation were all below $15 \%$. 


\section{Quadrant Compound Dose $(\mu \mathrm{M})$ nuOXPHOS mtOXPHOS}

$\begin{array}{lllll}\text { I } & \text { mebendazole } & 1 & 1.48 \pm 0.11 & 1.45 \pm 0.17\end{array}$

I deoxysappanone B $5 \quad 1.56 \pm 0.13 \quad 1.41 \pm 0.19$

II thimerosal

III perphenazine cycloheximide

Figure 4. Mining the compendium for small molecules that boost OXPHOS gene expression and decrease ROS levels

We used two complementary strategies to identify compounds in the screening compendium that elevate OXPHOS expression while suppressing ROS generation. (a) Mining the compendium for sets of structurally related compounds that boost OXPHOS expression and drop ROS levels. All compounds were organized into 624 clusters based on several chemical descriptors (molecular weight, $\log P$, number of hydrogen bond donors and acceptors, and number of rotatable bonds), and Mann-Whitney rank sum statistics for each cluster and each assay were calculated. The significance of each cluster in each assay is shown, with points above zero indicating positive scores, and points below zero showing negative composite scores. A nominal $p=0.01$ is delimited by the dashed lines, and the shaded area indicates the noise envelope. The red data points spotlight a single cluster that is significant for increased OXPHOS gene expression and decreased ROS. The chemical scaffold shared amongst the members of this cluster is shown in red. (b) Mining the compendium for individual compounds that give rise to desired assay profiles. We queried the compendium for compounds that boost OXPHOS expression and drop ROS levels. The distribution of ROS scores are shown for all compounds (gray), as well as for compounds associated with the highest OXPHOS gene expression (black). The latter follow a bimodal distribution, and the smaller mode (bracketed) contains six compounds that elevate OXPHOS expression and decrease ROS levels. The chemical structures of the compounds with the greatest decrease in ROS generation are shown. Five of these six compounds are known to be inhibitors of microtubules. 
$\frac{1}{0}$

\section{viability ATP}

MTT

$\Delta \Psi$

ROS

\section{atorvastatin pravastatin rosuvastatin}

\section{fluvastatin lovastatin simvastatin}

Figure 5. Secondary analyses of the effects of microtubule inhibitors on OXPHOS gene expression and physiology

(a) Compounds indicated in Figure $4 \mathrm{~b}$ were re-tested at $20 \mathrm{nM}, 200 \mathrm{nM}, 2 \mu \mathrm{M}$, and $20 \mu \mathrm{M}$.

Row-normalized gene expression levels of each gene are represented as a heat map. Beneath the heat map, dose-response analysis of ROS generation and viability are provided. No significant decrease in cell viability was observed (shaded area indicates the noise envelope, where $p>0.05$ ). All data shown are the results of four biological replicates per concentration. (b) mtDNA:nuDNA copy number analysis following treatment with four of the compounds ("deo", deoxysappanone B; "meb", mebendazole; "noc", nocodazole; "pac", paclitaxel), using quantitative PCR analysis of three biological replicates. Data are expressed 
as the fold increase in copy number relative to DMSO treatment alone. (c) Quantitative PCR measurement of PGC-1a gene expression, in response to either DMSO alone ("Con"), $5 \mu \mathrm{M}$ deoxysappanone B (“deo"), or $1 \mu \mathrm{M}$ mebendazole ("meb"). (d) Quantitative PCR measurement of the nuclear OXPHOS gene ATP5a1. Cells were either treated with compound alone (black bars) or in combination with $5 \mu \mathrm{M}$ of the ERRa inverse agonist XCT790 (gray bars). (e) Quantitative PCR measurement of the ROS scavenger MnSOD, as in ' $d$ '. Means and standard deviations of expression data are the result of four biological replicates. 


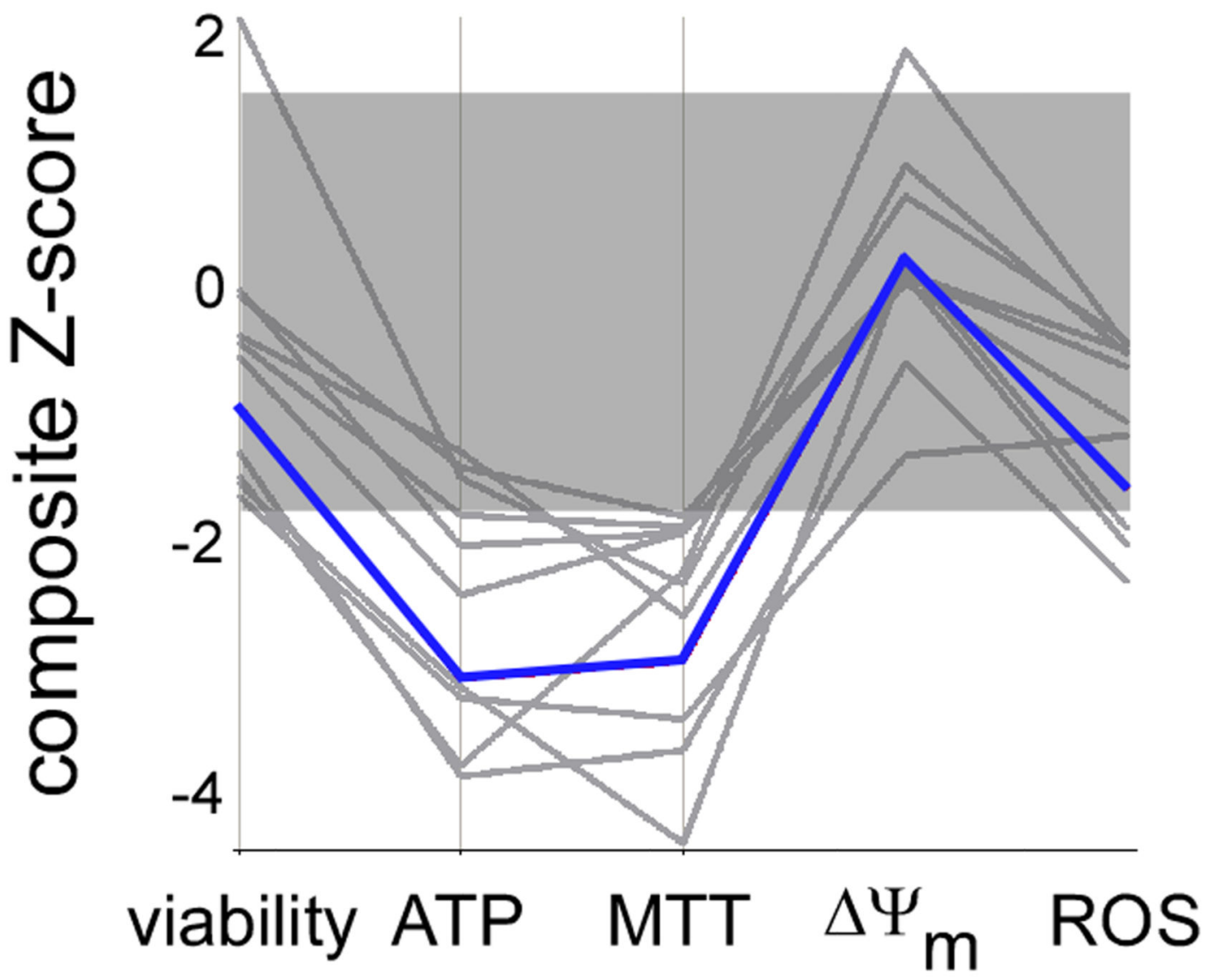

centroid statin score

10 nearest drugs (fluvastatin, lovastatin, simvastatin)

Figure 6. 

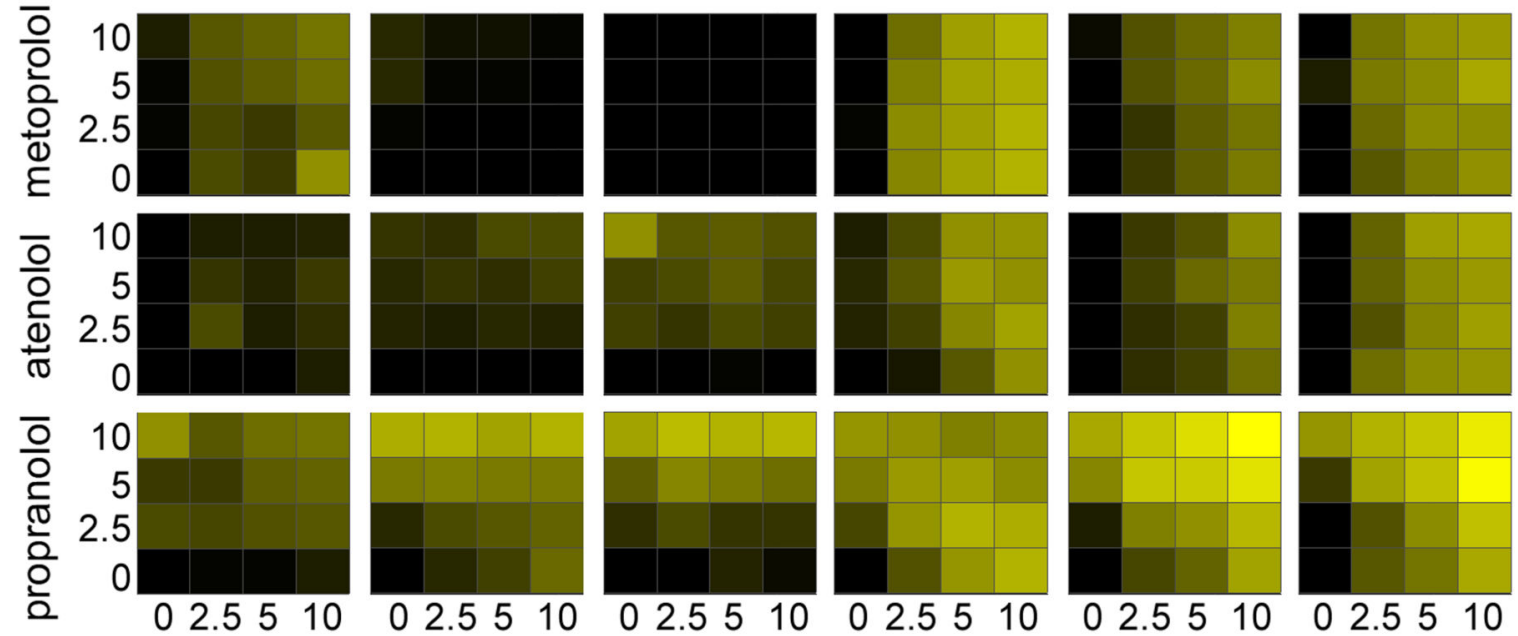
atorvastatin pravastatin rosuvastatin fluvastatin

Figure 7. 


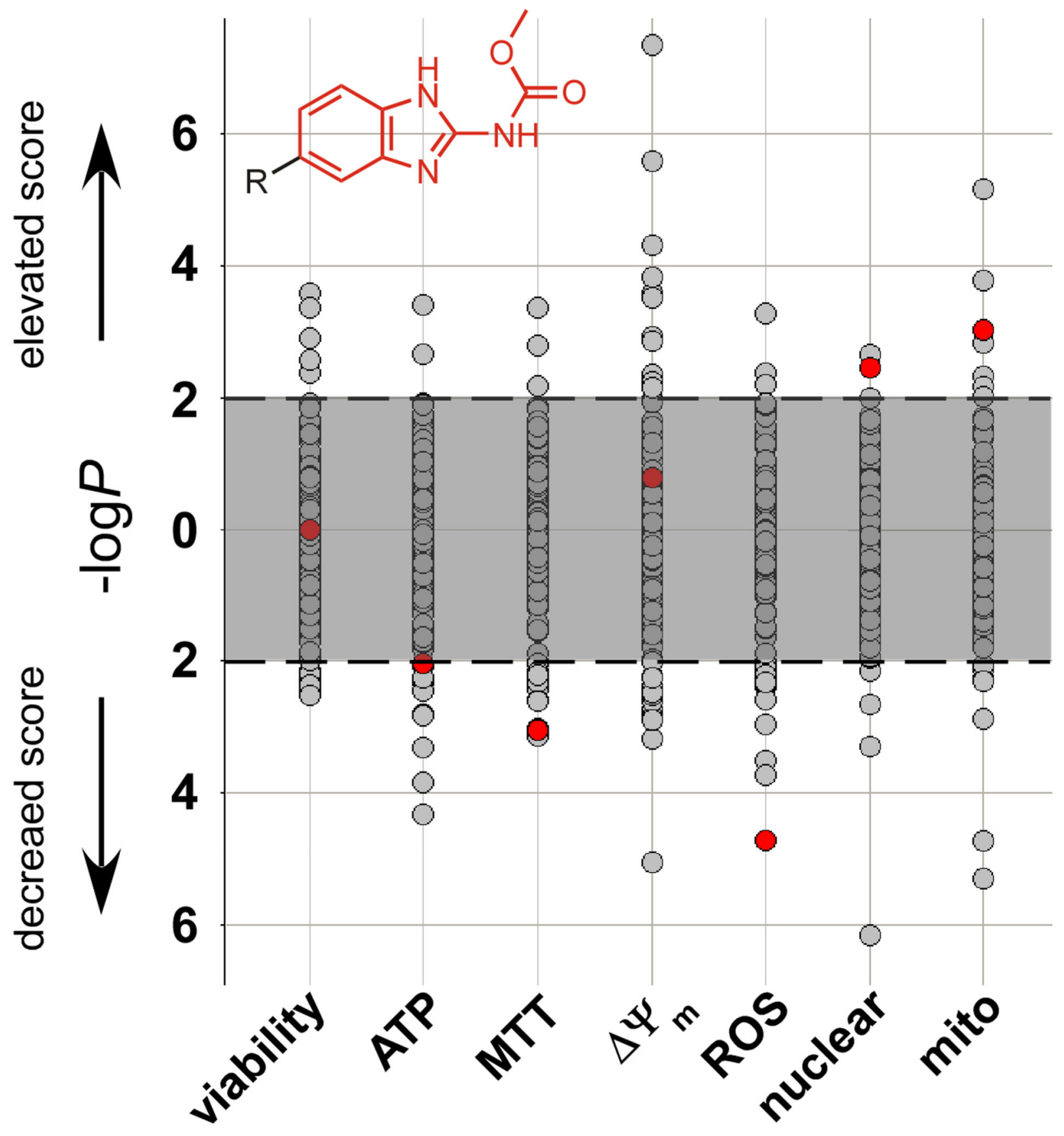

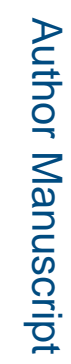

Figure 8. 


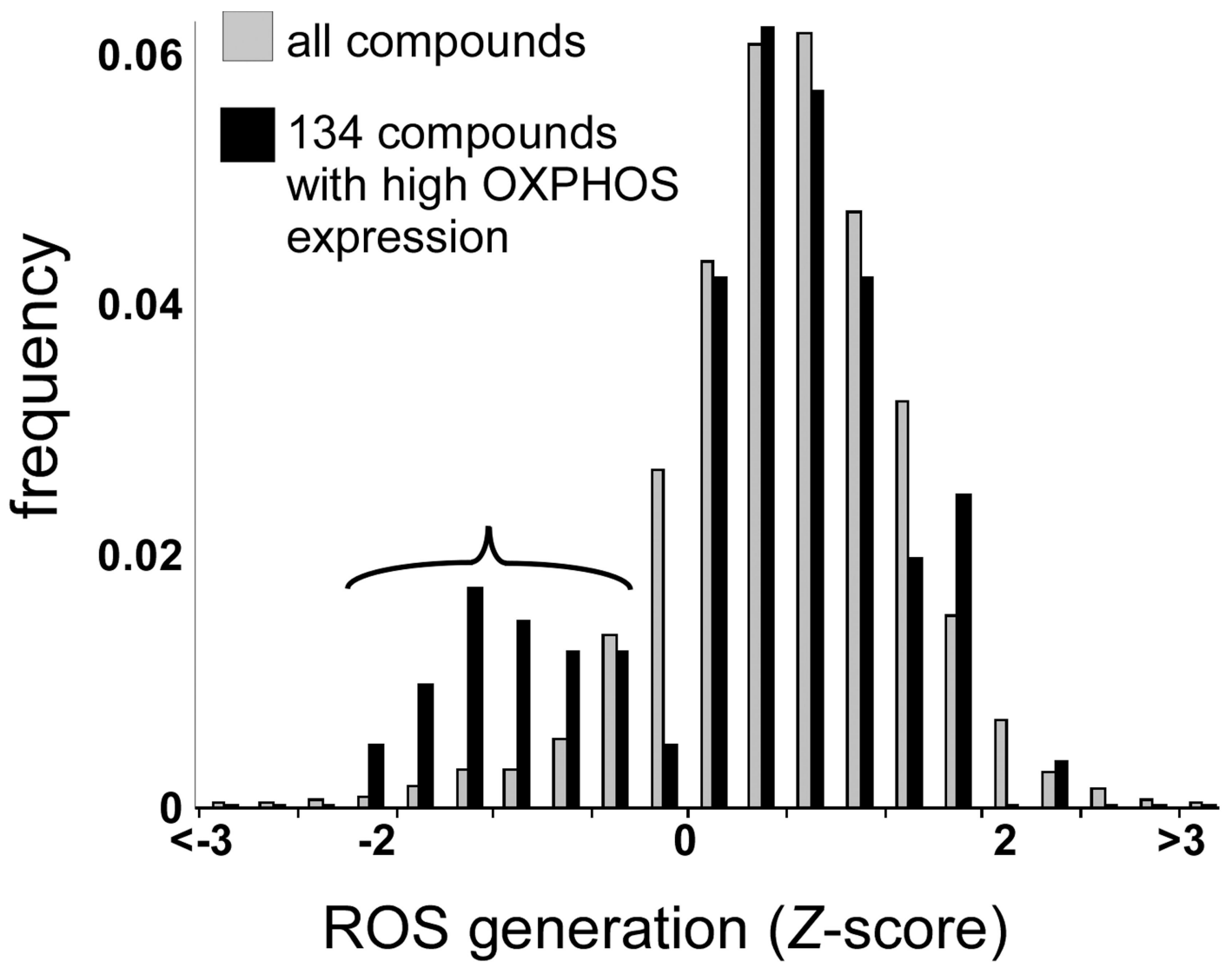

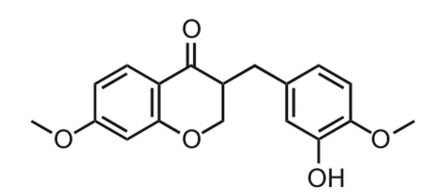
deoxysappanone

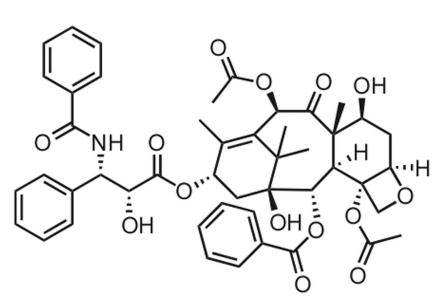

paclitaxel

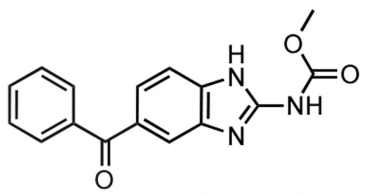

mebendazole

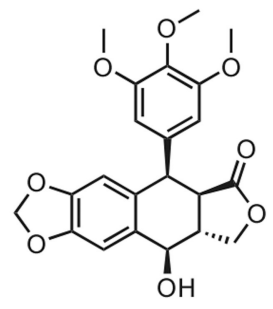

podofilox

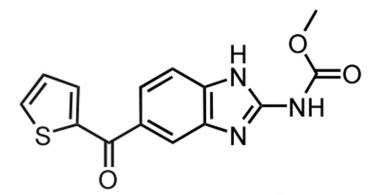

nocodazole

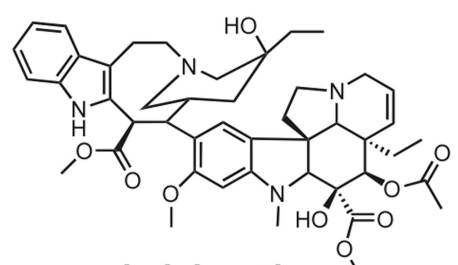

vinblastine

Figure 9. 


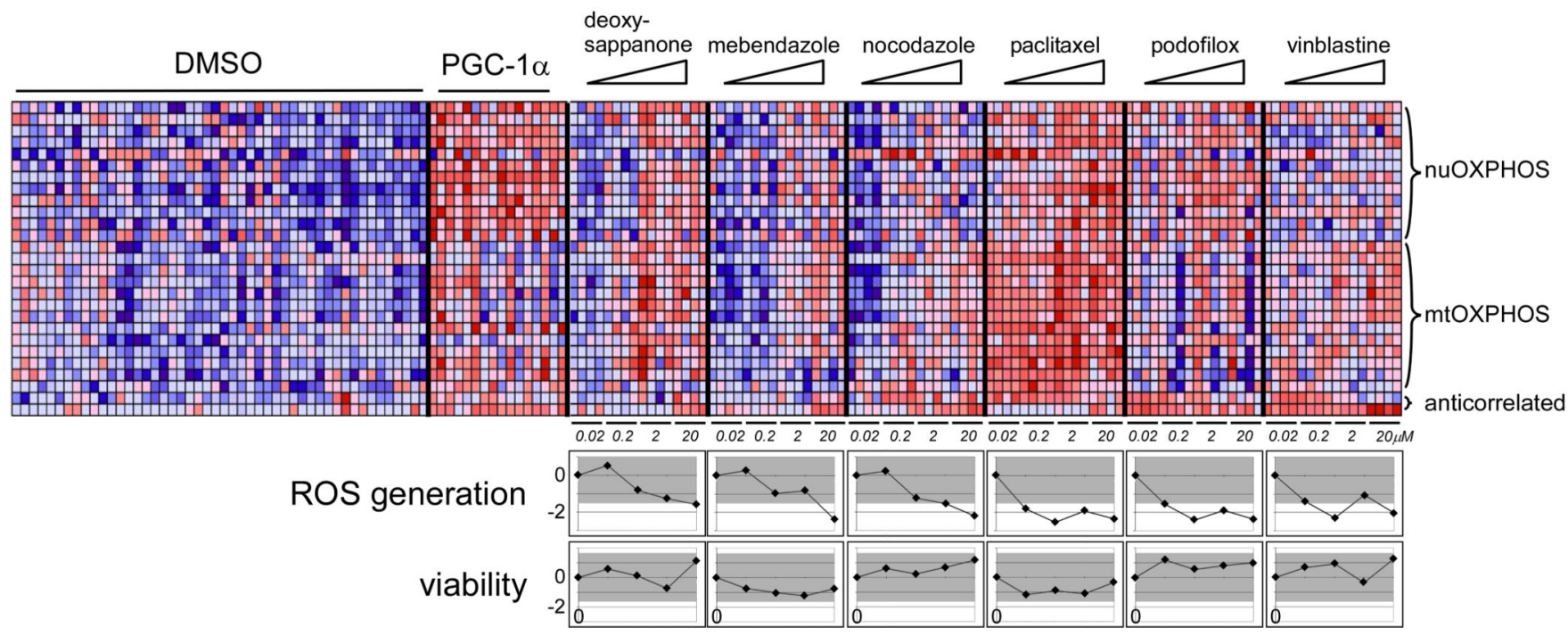

Figure 10. 

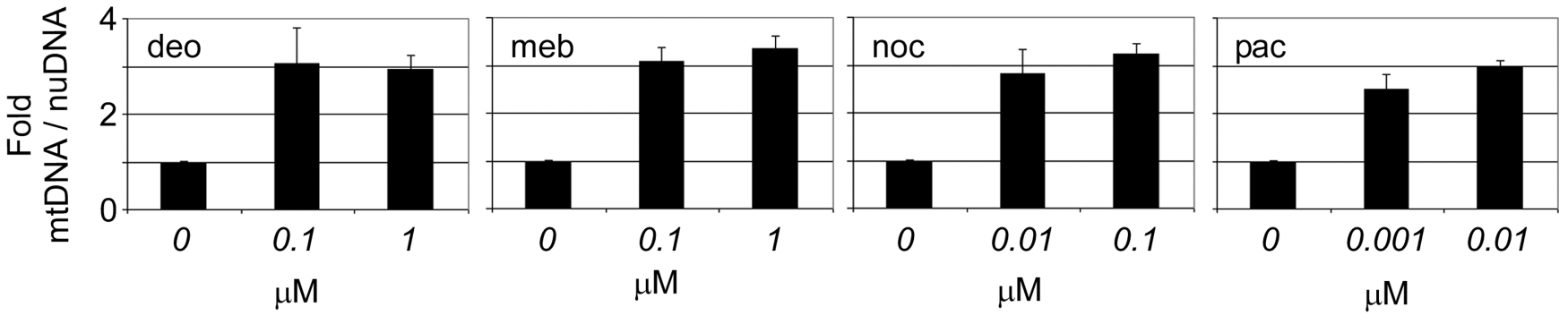

Figure 11. 


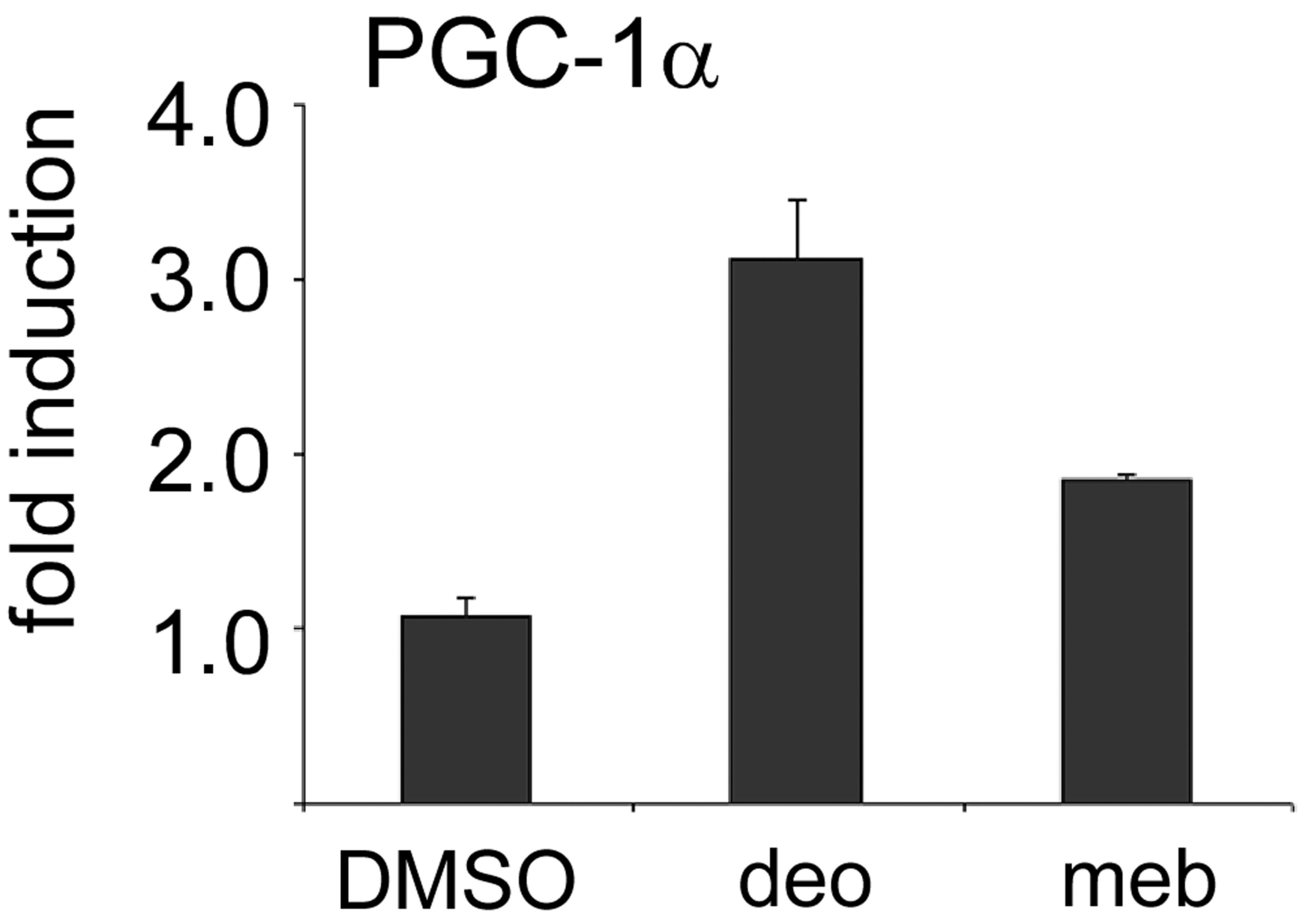

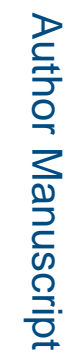

Figure 12.

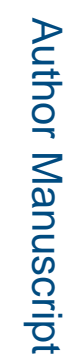




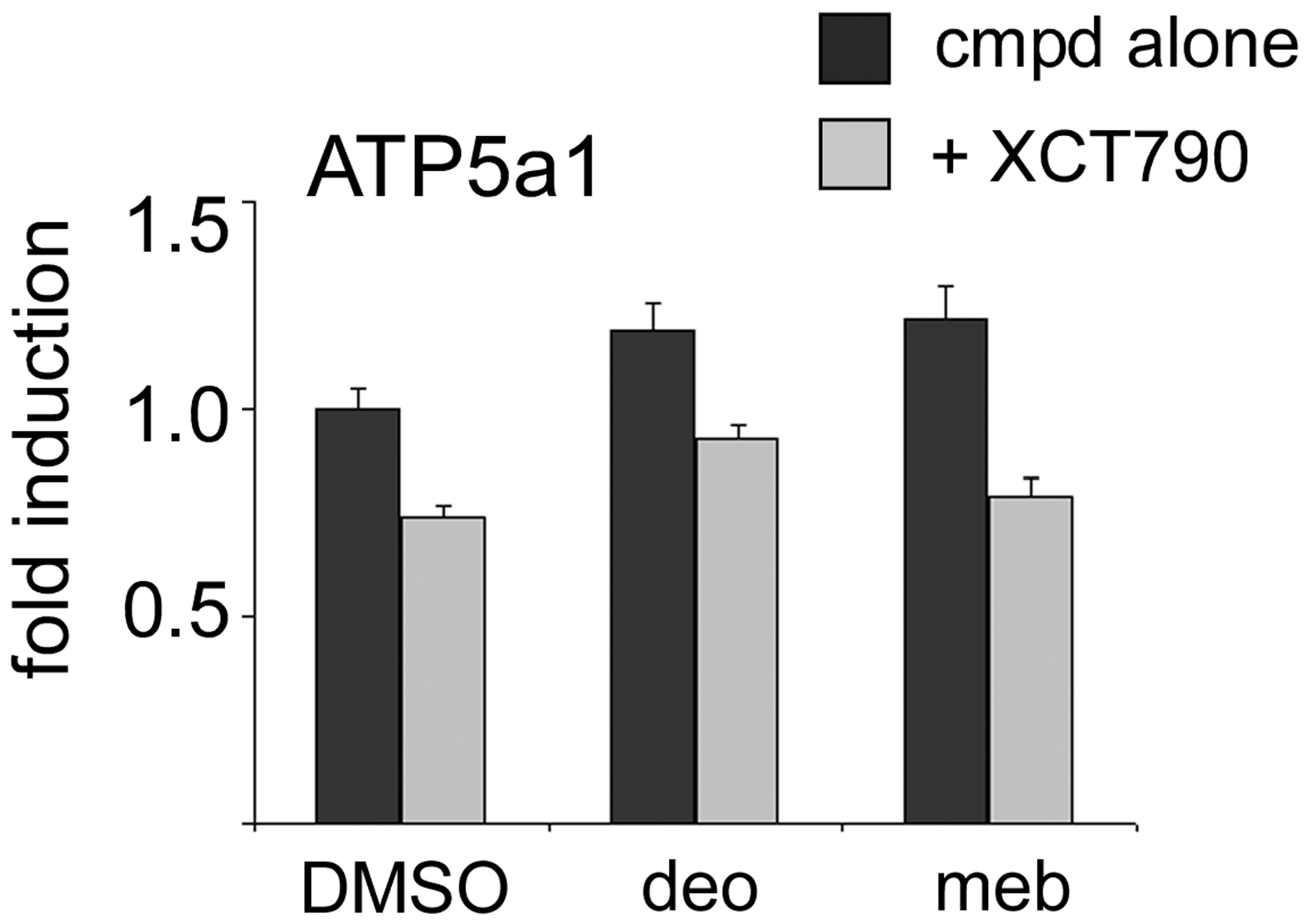

Figure 13. 


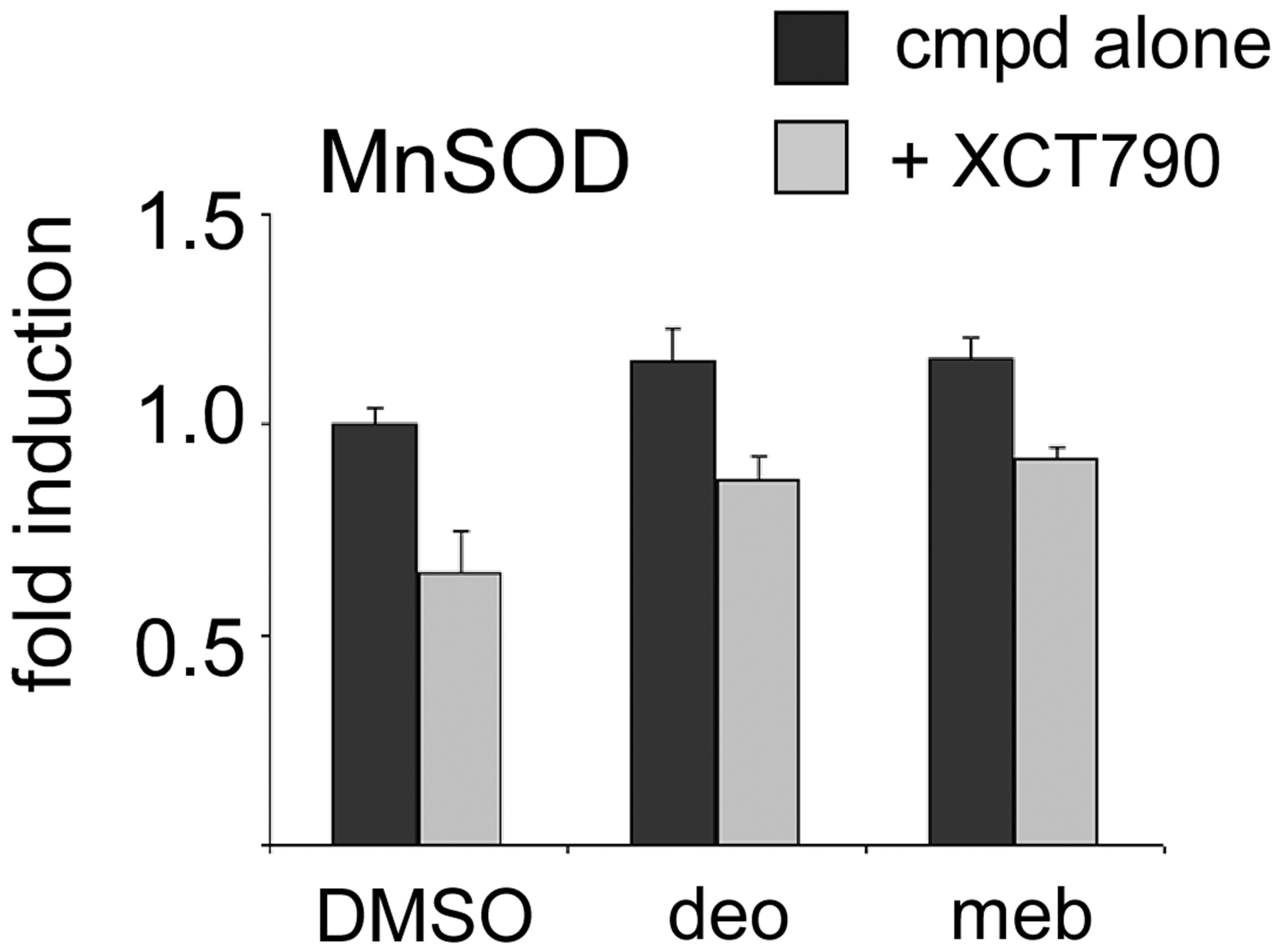

Figure 14. 